\section{Avaliação de campanhas de saúde com ênfase na sífilis congênita: uma revisão sistemática}

\author{
Evaluation of health campaigns with \\ emphasis on congenital syphilis: a \\ systematic review
}

Valéria Saraceni 1

Maria do Carmo Leal 2

Zulmira Maria de Araújo Hartz 3

\begin{abstract}
Campaigns are healthcare public policies means frequently used in Brazil, to educate, motivate or achieve population and/or healthcare professionals in relevant public health actions. This review pursued the gathering of input to assess health campaigns, specifically the methodologies used, focusing on congenital syphilis control campaigns. Keywords used were "congenital syphilis + assessment", "health campaigns + assessment" and, "campaign+ health+ assessment". Databases surveyed were Medline (Medical Literature Analysis and Retrieval System Online) of National Library of Medicine, LILACS (Latin American and Caribbean Health Sciences) and PAHO (Pan-American Health Organization), from 1980 to 2003. Thirty seven articles in this review, 15 related to congenital syphilis and assessment and 22 to healthcare campaigns and assessment. Review accomplished indicated a gap to be filled in this field specially related to methodology design. Notwithstanding the word "assessment" inclusion in these articles, the theoretical rationale determined was not consistent with the submitted proposals.
\end{abstract}

Key words Syphilis, congenital, Evaluation

\section{Resumo}

Campanhas têm sido instrumentos de políticas públicas de saúde, freqüentemente utilizadas no Brasil para esclarecer, motivar ou conseguir o apoio da população elou dos profissionais de saúde, em ações relevantes para a saúde pública. Esta revisão procurou levantar subsídios para a avaliação de campanhas de saúde, particularizando-se as metodologias utilizadas, tendo em vista a avaliação de campanhas dirigidas ao controle da sífilis congênita. Os conjuntos de palavraschave utilizados foram "sífilis congênita + avaliação"; "campanhas de saúde + avaliação" e, "campanha + saúde + avaliação". As bases de dados pesquisadas foram a Medline (Medical Literature Analysis and Retrieval System Online) da National Library of Medicine (EUA), a LILACS (Literatura LatinoAmericana e do Caribe em Ciências da Saúde) e da PAHO (Pan-American Health Organization), no período de 1980 e 2003. Foram incluídos 37 artigos nessa revisão, sendo 15 referentes à sífilis congênita e avaliação e 22 à campanha de saúde e avaliação. A revisão realizada mostrou que existe um espaço a ser preenchido neste campo, principalmente no que se refere à construção metodológica. Apesar da palavra avaliação constar nesses vários artigos, a fundamentação teórica encontrada não foi totalmente condizente com as propostas apresentadas.

Palavras-chave Sífilis congênita, Avaliação, Promoção da saúde 


\section{Introdução}

Campanhas têm sido instrumentos de políticas públicas de saúde, freqüentemente utilizadas no Brasil para esclarecer, motivar ou conseguir o apoio da população e/ou dos profissionais de saúde, em ações consideradas relevantes para a saúde pública. Em vários locais foram inicialmente utilizadas na área das doenças infecciosas e, posteriormente, no campo das doenças crônicas não transmissíveis. ${ }^{1}$

Contudo, a avaliação de campanhas poucas vezes é efetuada, e não se encontra metodologia clara e objetiva para tal fim. Na maior parte das vezes, os resultados de campanhas são expressos apenas em termos de cobertura e/ou população atingida. Nem mesmo os padrões de mudança epidemiológica que por ventura ocorram são conhecidos, embora sejam esperados pelas ações da campanha.

Em geral, as campanhas não estão estruturadas para responder questões relativas ao seu custo ou à sua efetividade, uma vez que os resultados de curto, médio e longo prazos não estão definidos. Elas são utilizadas para provocar uma movimentação momentânea, em determinado patamar, criando "o fato" e uma demanda real, porém não apresentando compromissos com desdobramentos que deveriam ser esperados, mas que não foram sequer planejados para acontecer. E, sem planejamento, também não são construídos indicadores ou apresentadas metas factíveis que permitam o monitoramento das atividades e a posterior avaliação.

Campanhas são instrumentos capazes de produzir transformações nas condições de saúde. A pertinência de sua avaliação resulta tanto na compreensão da política de saúde em geral quanto do seu alcance.

A revisão sistemática aqui apresentada procurou levantar, na literatura científica, subsídios para a avaliação de campanhas de saúde, particularizandose as metodologias utilizadas no processo de avaliação, dependentes do tipo de campanha realizada, por exemplo, de mídia, educativa, de ação ou de adesão.

A motivação para essa revisão veio da necessidade de avaliação de campanhas que tiveram a pretensão de dar visibilidade à sífilis congênita (SC). A persistência da SC no Município do Rio de Janeiro (MRJ), que sequer pode ser chamada de eventosentinela, tal a sua magnitude, se deve ao fato de que as atividades básicas e de baixo custo necessárias à eliminação da doença realizadas nas ações de rotina da assistência pré-natal não mudavam o cenário. As campanhas para eliminação da SC realizadas em
1999 e 2000 foram entendidas como possíveis agentes transformadores desse cenário. ${ }^{2}$

Apesar da importância do agravo, é pequena a quantidade de trabalhos publicados no país enfocando estratégias para a sua eliminação, meta apregoada, mas ainda não alcançada. O Ministério da Saúde havia estabelecido uma meta de menos de um caso de SC por 1000 nascidos vivos no ano 2000, em acordo firmado com a Organização Pan-Americana de Saúde.3,4

A campanha pretendia alcançar a população e os profissionais de saúde, em atividades de informação e educação, obtidas tanto através da mídia, como através de capacitações e atividades de educação em saúde. O pressuposto teórico de base, enfocando-se a população, era aumentar a visibilidade e o conhecimento da doença, bem como possibilitar, aos profissionais de saúde, intervir de maneira mais efetiva em numa prática assistencial que não apresentava resultados satisfatórios. 5,6

\section{Um pouco da história da sífilis e das suas campanhas}

A sífilis é uma doença antiga, com mais de 500 anos de existência. Relatos de sua presença na Europa logo após o descobrimento da América mesclam-se com a sua existência no Velho Continente em período anterior às viagens de Cristóvão Colombo ao novo mundo. 7 A sífilis transformou-se em uma pandemia, com um quadro clínico muito agudo, freqüentemente fatal no estágio secundário, descrita por muitos autores na passagem para o século XVI. 8

$\mathrm{O}$ agente etiológico da sífilis foi descoberto por Fritz Richard Schaudinn e Paul Erich Hoffman em 1905 e foi denominado Spirochaeta pallida. O primeiro teste sorológico para a doença tornou-se disponível em 1906, através de Wassermann, Neisser e Bruck, utilizando a técnica de fixação de complemento. $\mathrm{O}$ antígeno para sua reação foi preparado a partir do extrato hepático de um natimorto de mãe com sífilis. ${ }^{9}$

A transmissão do agente se dá de pessoa a pessoa, durante o contato sexual, na maior parte dos casos. Pode ocorrer transmissão através da transfusão de sangue contaminado, pelo contato direto com lesões cutâneo-mucosas infectantes, por via transplacentária para o feto e contaminação do último no canal de parto. A transmissão ocupacional é rara. O risco de transmissão por parceiro sexual está estimado em $60 \% .10$

As primeiras descrições da sífilis congênita são atribuídas a Lopez de Villalobos e Fracastoro. 
Paracelsus parece ter sido o primeiro a aventar a transmissão in utero. Sir Jonathan Hutchinson é a personalidade do meio acadêmico mais ligada à sífilis congênita por sua descrição da tríade de Hutchinson, com a má-formação dos dentes que recebeu seu nome, a ceratite intersticial e a surdez neuro-sensorial, por lesão do oitavo par craniano. ${ }^{11}$

A evolução do tratamento da sífilis foi lenta a partir dos compostos mercuriais. Em 1910, Paul Erlich trouxe para a prática clínica um composto de arsênico, a arsfenamina, conhecida como composto número 606 ou salvarsan. As pesquisas subseqüentes sobre os compostos arsênicos levaram ao composto número 914 ou neosalvarsan. A introdução da penicilina para o tratamento da sífilis na década de 40 do século XX modificou o panorama. 12 Logo após a introdução dessa droga como terapia, as taxas de sífilis começaram a diminuir nos EUA, incluindo as taxas da sífilis congênita. 11

As campanhas contra as doenças venéreas, em especial contra a sífilis, iniciaram-se na virada para o século XX. Fournier lançou na França o primeiro esforço concentrado médico-social, com a criação da Sociedade Francesa de Profilaxia Moral em 1900. Nos Estados Unidos da América, Prince A. Morrow fundou a Sociedade para a Profilaxia Moral e Sanitária em 1905. As descobertas do agente etiológico, do teste sorológico e do medicamento salvarsan construíram a base científica para tal. ${ }^{1}$

No Brasil, a mesma sociedade é criada em 1901, mas não floresce tanto quanto as estrangeiras. Tal não se deu por falta de movimentação dos sifilógrafos nacionais, pois na entrada do século $\mathrm{XX}$, compartilhavam a idéia em voga de ser a sífilis a causa de quase todos os males e eram detentores do poder na medicina, como pode ser visto através de citação atribuída a Sir William Osler "He who knows syphilis, knows medicine". 11

Em seu livro "Tributo à Vênus: a luta contra a sífilis no Brasil, da passagem do século aos anos 40", Carrara 13 faz excelente revisão do assunto, de onde se destaca a criação da Inspetoria de Profilaxia da Lepra e das Doenças Venéreas, em 1920, que institui a primeira campanha contra a sífilis no país, e, também, a I Conferência Nacional de Defesa contra a Sífilis, realizada em 1940.

\section{Métodos}

Neste trabalho foram revistas as bases de dados Medline da National Library of Medicine (EUA), a LILACS (Literatura Latino-Americana e do Caribe em Ciências da Saúde) e da PAHO (Pan-American
Health Organization), no período de 1980 e 2003. Embora tenha-se ampliado a busca para antes de 1980, um único trabalho anterior a essa data foi incluído pela sua relevância com a busca. Outros trabalhos que constavam nas listas de referências dos artigos encontrados foram algumas vezes utilizados como leitura complementar.

Os conjuntos de palavras-chave utilizados foram "sífilis congênita + avaliação"; congenital syphilis + evaluation; "campanhas de saúde + avaliação"; health campaign + evaluation; "campanha + saúde + avaliação" e health + campaign + evaluation.

A palavra-chave com maior capacidade de discriminação entre os estudos selecionados foi "avaliação", excluindo-se a avaliação clínica dos casos de sífilis materna ou de SC e a avaliação de testes laboratoriais para diagnóstico de sífilis. Partiuse do princípio de que a sífilis na gestante tem critérios diagnósticos bem definidos, de tecnologia simples e de baixo custo, e que o tratamento disponível na rede pública está de acordo com os padrões estabelecidos internacionalmente.14-17 As normas para a eliminação da SC no Brasil estão claramente descritas pelo Ministério da Saúde, 18 e são semelhantes às normas dos Centers for Disease Control and Prevention (CDC). 19

A "avaliação" foi aqui entendida como um estudo com uma metodologia clara, que permita dizer da efetividade de uma intervenção sobre uma situação definida, ou mesmo da eficácia de um medicamento no tratamento da sífilis materna, evitando o agravo em tela. Esta última questão, da eficácia do tratamento, foi mantida por ser a penicilina considerada o padrão-ouro do tratamento da grávida até o momento.

Os resultados obtidos na busca encontram-se na Tabela 1. Usando-se as ferramentas de busca do PubMed da National Library of Medicine, EUA, e da Biblioteca Virtual em Saúde (BVS) da PAHO, foram encontradas um total de 220 referências. Foram excluídas as referências que não possuíam abstracts; as referências de artigos originais que se encontravam em línguas que não o inglês, francês, português ou espanhol; os relatos de casos clínicos; as cartas ao editor; os artigos de revisão; os artigos de avaliação clínica e os de avaliação laboratorial da doença; e, os que não apresentavam resultados, os quais estavam prometidos para futuros artigos. Embora sempre aparecesse nas buscas, o artigo de Saraceni e Leal,2 intitulado "Avaliação da efetividade das campanhas de eliminação da sífilis congênita no município do Rio de Janeiro, 1999 e 2000" não foi incluído, uma vez que essa revisão sistemática é parte da dissertação de doutorado de 
Tabela 1

Resultados da busca em base de dados bibliográficos, 1979-2003.

\begin{tabular}{lrrr}
\hline Palavras-chave & Bases & Total & Seleção \\
\hline Sífilis congênita + avaliação & Lilacs & 8 & 3 \\
Congenital syphilis + evaluation & Medline, PAHO & 83 & 12 \\
Campanha de saúde + avaliação & Lilacs & 16 & 4 \\
Health campaign + evaluation & Medline, PAHO & 18 & 8 \\
Campanha + saúde + avaliação & Lilacs & 0 & 0 \\
Health + campaign + evaluation & Medline, PAHO & 95 & 10 \\
Total & & 220 & 37 \\
\end{tabular}

Medline $=$ Medical Literature Analysis and Retrieval System Online; Lilacs = Literatura Latino Americana e do Caribe em Ciências da Saúde; PAHO = PanAmericana Health Organization.

um dos autores, assim como o próprio artigo também o é.

\section{Resultados}

Foram incluídos 37 artigos nesta revisão, sendo 30 encontrados no Medline, sete no LILACS e nenhum na PAHO. Quinze artigos são referentes à sífilis congênita e avaliação e 22 artigos à campanhas de saúde e avaliação.

Na Tabela 2 encontram-se os tipos de estudo e a medida de desfecho utilizada na avaliação. Como pode ser verificada, a maioria dos estudos utilizou medidas epidemiológicas para avaliar o resultado de suas estratégias. Outros estudos utilizaram técnicas qualitativas.

Em relação à avaliação e sífilis congênita, a maioria dos estudos $(53,4 \%)$ fez uma avaliação em termos de vigilância epidemiológica (VE). Tayra 20 preocupou-se com a incidência da SC no local estudado, sem discutir a confiabilidade dos dados ou estimar a subnotificação. Silva Jr. et al. ${ }^{21}$ realizaram o cruzamento dos dados das bases de dados do Sistema Nacional de Agravos de Notificação (SINAN) e do Sistema de Informações Hospitalares (SIH) do Sistema Único de Saúde (SUS), verificando um descompasso entre o número de internações registradas e o número de casos de SC notificados. Em Cuba, foi realizada a avaliação do sistema de VE22 através de entrevistas semi-estruturadas e observação não participativa com médicos e enfermeiras do sistema de medicina de família, revelando uma baixa capacitação desses profissionais em relação à VE. Recorrendo à revisão de prontuários, encontrou-se uma baixa suspeição dos casos de SC

Tabela 2

Tipo de estudo e medida de desfecho utilizada na avaliação dos artigos.

\begin{tabular}{|c|c|c|}
\hline \multicolumn{3}{|c|}{ Sífilis congênita + avaliação } \\
\hline Tipo de estudo & Número de artigos & Medida do desfecho \\
\hline Avaliação da vigilância epidemiológica (VE) & 6 & Incidência de SC \\
\hline Avaliação de profissionais de saúde sobre VE & 1 & Descrição qualitativa \\
\hline Avaliação da eficácia do tratamento da sífilis materna & 3 & Eficácia de medicamento \\
\hline Avaliação da eficiência do sistema de screening & 5 & Prevalência de sífilis, incidência de SC \\
\hline \multicolumn{3}{|c|}{ Campanha de saúde + avaliação } \\
\hline Tipo de estudo & Número de artigos & Medida do desfecho \\
\hline Eficácia do tratamento de DST na prevenção do HIV & 2 & Incidência de DST / HIV \\
\hline Campanha educativa sobre HIV para a população geral & 1 & Incidência de sífilis e de HIV \\
\hline Campanha de mídia para população e profissionais de saúde & 5 & Prevalência de sífilis \\
\hline Profissionais de saúde e VE, busca de parceiros, custo-efetividade & 1 & $\begin{array}{l}\text { Incidência de sífilis, análise de custo- } \\
\text { efetividade }\end{array}$ \\
\hline Campanha de mídia para a população geral & 6 & Descrição qualitativa \\
\hline Campanha para profissionais de saúde & 1 & Descrição qualitativa \\
\hline Caso controle sobre vacina & 1 & Odds ratio \\
\hline Pesquisa operacional & 1 & Efetividade de intervenção em serviço \\
\hline Efetividade de intervenção em saúde & 4 & Incidência do agravo \\
\hline
\end{tabular}

DST = Doenças sexualmente transmisíveis; VE = Vigilância epidemológica; SC = Sífilis congênita 
por parte dos médicos no estado de Minnesota, EUA. ${ }^{23}$. Uma avaliação dos CDC publicada em 1995 para o Estado de New Jersey, EUA, mostrou que $20 \%$ dos casos de SC estavam mal classificados como não-casos, o que os levou a considerar a VE local não preparada para lidar com o agravo. 24. Para avaliar a efetividade relativa do aumento da oferta de testes e incremento da VE, em uma situação de aumento do número de casos de sífilis, comparou-se a curva de incidência de SC da cidade de Los Angeles com a de outras cidades, observando-se um retorno ao nível basal de incidência naquela cidade após a intervenção, diferente do comportamento da curva das outras, onde nenhum esforço adicional foi realizado.25. Mascola et al. ${ }^{26}$ concluíram pela subnotificação de casos no Texas, EUA, após a revisão de 50 casos notificados no ano de 1982, usando a proporção de casos sintomáticos $(62 \%)$ como indicador, valor acima do estimado para o global das notificações de SC pela revisão da literatura.

Os três estudos que avaliaram a eficácia do tratamento da sífilis materna com o uso da penicilina benzatina nas doses preconizadas tiveram bons resultados,27-29 em consonância com a revisão sistemática que consta na Biblioteca Cochrane sobre o assunto.17. A efetividade do screening da sífilis na gestação e no parto foi avaliada em quatro estudos, ressaltando a importância de ser realizado, mesmo que tardiamente no momento do parto. Nesse momento, a oportunidade já está perdida para a prevenção da SC. Todavia, ainda existe a chance de tratar o recém-nascido, reduzindo-se a probabilidade de danos futuros. ${ }^{30-33}$ Fitzgerald et al. ${ }^{34}$ publicaram recentemente uma nova avaliação de sugestão de descentralização do screening, de modo a torná-lo mais efetivo.

Para os artigos encontrados através da busca de palavras-chave "avaliação + campanha + saúde", dois referiam-se à eficácia do tratamento de doenças sexualmente transmissíveis (DST) como forma de prevenção da aquisição da infecção pelo HIV.35-36 As prevalências de DST e de HIV foram mensuradas antes e depois da intervenção.

Outro artigo versou sobre a avaliação de campanha informativa sobre HIV na mídia em Cuba. 37 Nesse país realizava-se uma testagem mandatória, após a qual os portadores do HIV eram recolhidos a uma quarentena especial pelas autoridades de saúde, deixando transparecer aos cidadãos locais uma certa segurança em relação à transmissão do HIV. Ao final da campanha, embora tenha ocorrido uma diminuição da incidência de HIV, evidenciou-se um aumento da incidência de sífilis.

Campanhas de mídia para a população e para os profissionais de saúde, com outras atividades, estavam presentes em cinco artigos, $38-42$ onde a avaliação lançou mão de estudos transversais (surveys) e medidas de incidência da sífilis. Um outro artigo, além de descrever uma campanha envolvendo a população em geral e os profissionais de saúde, apresentou um estudo de custo-efetividade para a busca de parceiros de indivíduos com sífilis infectante (sífilis primária, secundária e latente precoce), concluindo que a intensificação da busca de contactantes não trouxe acréscimo ao número total de casos identificados, tendo, porém, aumentado os custos. 43 Esse artigo fala do envolvimento de redes sociais nas intervenções realizadas como forma mais contundente de atingir a população-alvo.

Realização de campanha de mídia voltada apenas para a população em geral foi motivo de seis artigos, cobrindo diversos campos do conhecimento, como a AIDS,44-45 um estudo sobre canais de veiculação de campanhas de saúde, 46 o melanoma cutâneo, 47 a saúde oral 48 e a oftalmologia preventiva. 49 As metodologias utilizadas na avaliação foram os estudos transversais, com estudos de prevalência e a realização de enquetes. Apenas um artigo teve como alvo somente os profissionais de saúde, no caso em campanha sobre saúde oral50 avaliando o conhecimento, atitudes e comportamento dos dentistas frente à doença periodontal, através de uma amostra desses respondendo a um questionário por correio.

Um único trabalho desenhou sua avaliação como pesquisa operacional,51 com ênfase no acompanhamento de resultados, avaliando a eficácia e a eficiência das intervenções e do serviço oferecido. Outro artigo usou a metodologia de estudo casocontrole para avaliar a eficácia de vacina antimeningocócica BC no Rio de Janeiro. 52

Os quatro artigos restantes lidaram com a efetividade de intervenções variadas em saúde.53-56 Valendo-se de grupos de comparação em estudos de prevalência ou através de inquéritos.

A descrição da metodologia utilizada em cada artigo encontra-se na Tabela 3. 
Tabela 3

Correlação entre autores, metodologia e medidas de desfecho.

\begin{tabular}{|c|c|c|c|}
\hline \multicolumn{4}{|c|}{ Sífilis congênita + avaliação } \\
\hline $\begin{array}{l}\text { Primeiro autor, } \\
\text { ano de publicação }\end{array}$ & Metodologia utilizada & Medida do desfecho & Tipo de estudo \\
\hline Tayra; 2001.20 & Incidência de SC, a partir dos casos notificados no SINAN & Incidência de SC & $\begin{array}{l}\text { Estudo de base } \\
\text { pulacional }\end{array}$ \\
\hline Silva Jr.et al.; 2000.21 & $\begin{array}{l}\text { Relacionamento de banco de dados do SINAN e SIH para verificar } \\
\text { possível subnotificação }\end{array}$ & Incidência de SC & $\begin{array}{l}\text { Estudo de base } \\
\text { pulacional }\end{array}$ \\
\hline Martin et al.; 2001.23 & $\begin{array}{l}\text { Revisão de prontuários para avaliar suspeição diagnóstica de SC pela } \\
\text { definição de caso }\end{array}$ & Incidência de SC & $\begin{array}{l}\text { Estudo transver- } \\
\text { sal }\end{array}$ \\
\hline CDC; 1995.24 & $\begin{array}{l}\text { Revisão de prontuários para avaliar suspeição diagnóstica de SC pela } \\
\text { definição de caso }\end{array}$ & Incidência de SC & $\begin{array}{l}\text { Estudo transver- } \\
\text { sal }\end{array}$ \\
\hline Cohen et al.; 1992.25 & $\begin{array}{l}\text { Avaliação de intervenção após aumento do número de casos de sífilis - } \\
\text { medida da incidência de SC antes e depois, comparando com grupo sem } \\
\text { intervenção }\end{array}$ & Incidência de SC & $\begin{array}{l}\text { Estudo observa- } \\
\text { cional }\end{array}$ \\
\hline Mascola et al.; 1985.26 & $\begin{array}{l}\text { Revisão de prontuários para avaliar suspeição diagnóstica de SC pela } \\
\text { definição de caso }\end{array}$ & Incidência de SC & $\begin{array}{l}\text { Estudo transver- } \\
\text { sal }\end{array}$ \\
\hline $\begin{array}{l}\text { Gomes de Haz e Farias } \\
\text { Reinoso; } 1998.22\end{array}$ & $\begin{array}{l}\text { Técnicas qualitativas de entrevistas semi-estruturadas e observação } \\
\text { não-participativa para avaliar qualificação dos profissionais de VE }\end{array}$ & $\begin{array}{l}\text { Grau de qualificação } \\
\text { profissional }\end{array}$ & $\begin{array}{l}\text { Eficácia de me- } \\
\text { dicamento }\end{array}$ \\
\hline $\begin{array}{l}\text { Phaosavasdi et al.; } \\
\text { 1989.29 }\end{array}$ & $\begin{array}{l}\text { Comparação de grupo tratado com penicilina benzatina, de acordo com } \\
\text { protocolo do } C D C \text {, com grupo não tratado }\end{array}$ & $\begin{array}{l}\text { Eficácia da penicilina } \\
\text { ben-zatina }\end{array}$ & $\begin{array}{l}\text { Eficácia de me- } \\
\text { dicamento }\end{array}$ \\
\hline $\begin{array}{l}\text { McFarlin e Bottoms; } \\
\text { 1995.28 }\end{array}$ & $\begin{array}{l}\text { Comparação de grupo tratado com penicilina benzatina, de acordo com } \\
\text { protocolo do CDC, com grupo não tratado }\end{array}$ & $\begin{array}{l}\text { Eficácia da penicilina } \\
\text { benzatina }\end{array}$ & $\begin{array}{l}\text { Eficácia de me- } \\
\text { dicamento }\end{array}$ \\
\hline $\begin{array}{l}\text { Alexander et al.; } \\
\text { 1999.27 }\end{array}$ & $\begin{array}{l}\text { Comparação de grupo tratado com penicilina benzatina, de acordo com } \\
\text { protocolo do } C D C \text {, com grupo não tratado }\end{array}$ & $\begin{array}{l}\text { Eficácia da penicilina } \\
\text { benzatina }\end{array}$ & $\begin{array}{l}\text { Estudo transver- } \\
\text { sal }\end{array}$ \\
\hline $\begin{array}{l}\text { Garland e Kelly; } \\
1989.32\end{array}$ & Efetividade do screening de sífilis durante a gestação & $\begin{array}{l}\text { Prevalência de sífilis } \\
\text { na gestação, inci- } \\
\text { dência de SC }\end{array}$ & $\begin{array}{l}\text { Estudo transver- } \\
\text { sal }\end{array}$ \\
\hline $\begin{array}{l}\text { Swingler e Van } \\
\text { Coeverden den de } \\
\text { Groot et al.; } 1993.33\end{array}$ & Efetividade do screening de sífilis durante a gestação & $\begin{array}{l}\text { Prevalência de sífilis } \\
\text { na gestação, incidên- } \\
\text { cia de SC }\end{array}$ & $\begin{array}{l}\text { Estudo transver- } \\
\text { sal }\end{array}$ \\
\hline Coles et al.; 1998.30 & Efetividade do screening de sífilis durante a gestação & $\begin{array}{l}\text { Prevalência de sífilis } \\
\text { na gestação, incidên- } \\
\text { cia de SC }\end{array}$ & $\begin{array}{l}\text { Estudo transver- } \\
\text { sal }\end{array}$ \\
\hline $\begin{array}{l}\text { Fitzgerald et al.; } 1998 \text { e } \\
2003.31,34\end{array}$ & Efetividade do screening de sífilis durante a gestação & $\begin{array}{l}\text { Prevalência de sífilis } \\
\text { na gestação, incidên- } \\
\text { cia de SC }\end{array}$ & $\begin{array}{l}\text { Estudo tranver- } \\
\text { sal }\end{array}$ \\
\hline
\end{tabular}


Campanha de saúde + avaliação

\begin{tabular}{|c|c|c|c|}
\hline $\begin{array}{l}\text { Primeiro autor, } \\
\text { ano de publicação }\end{array}$ & Metodologia utilizada & Medida do desfecho & Tipo de estudo \\
\hline Hayes; 1995.35 & $\begin{array}{l}\text { Comparação da incidência de DST e HIV após intervenção randomizada } \\
\text { de tratamento de DST em } 12 \text { comunidades na Tanzânia }\end{array}$ & $\begin{array}{l}\text { Incidência de DST e } \\
\text { HIV }\end{array}$ & $\begin{array}{l}\text { Estudo } \\
\text { transversal }\end{array}$ \\
\hline Perez-Stable; 1992.37 & $\begin{array}{l}\text { Avaliação da estratégia do governo cubano para redução da } \\
\text { transmissão do HIV, que, com a quarentena imposta aos HIV+, } \\
\text { conseguiu conter a infecção pelo HIV, mas não foi capaz de iminuir a } \\
\text { incidência de sífilis, apesar de estimular o uso de preservativo }\end{array}$ & $\begin{array}{l}\text { Incidência de sífilis e } \\
\text { de HIV }\end{array}$ & $\begin{array}{l}\text { Descrição } \\
\text { qualitativa, } \\
\text { estudo } \\
\text { transversal }\end{array}$ \\
\hline Towpik; 1979.42 & $\begin{array}{l}\text { Campanha para controle de DST que durou oito anos na Polônia, com } \\
\text { capacitação de profissionais, screening de massa, busca de parceiros }\end{array}$ & Incidência de sífilis & $\begin{array}{l}\text { Estudo } \\
\text { transversal }\end{array}$ \\
\hline $\begin{array}{l}\text { Bentley e Holloway; } \\
1993.38\end{array}$ & $\begin{array}{l}\text { Campanha de saúde oral para aumentar a captação de lactentes no } \\
\text { Reino Unido, cuja efetividade foi avaliada por verificação de crianças } \\
\text { que chegaram aos dentistas, a partir de listas de convocação aos pais, } \\
\text { efetuadas por facilitadores }\end{array}$ & $\begin{array}{l}\text { Aumento do acesso } \\
\text { aos serviços }\end{array}$ & $\begin{array}{l}\text { Estudo } \\
\text { transversal }\end{array}$ \\
\hline Card .39 & $\begin{array}{l}\text { Campanha de prevenção da gravidez na adolescência, embasada pela } \\
\text { experiência de prevenção à DST/AIDS, revisão das experiências } \\
\text { encontradas na literatura }\end{array}$ & $\begin{array}{l}\text { Impacto do conheci- } \\
\text { mento }\end{array}$ & $\begin{array}{l}\text { Estudo } \\
\text { transversal }\end{array}$ \\
\hline Storey et al.; 1999.41 & $\begin{array}{l}\text { Avaliação de dois programas de rádio comunitária no Nepal, ligados à } \\
\text { saúde reprodutiva, um para o público geral e um para profissionais de } \\
\text { saúde. Impacto avaliado através de painel de mulheres casadas, três } \\
\text { tempos de observação cliente-cuidador e entrevistas pós-consulta }\end{array}$ & $\begin{array}{l}\text { Impacto na saúde } \\
\text { reprodutiva }\end{array}$ & $\begin{array}{l}\text { Estudo } \\
\text { transversal }\end{array}$ \\
\hline Chen et al.; 2002.40 & $\begin{array}{l}\text { Avaliação de intervenção para contenção de surtos de sífilis, com } \\
\text { campanha de mídia, hotline, organização comunitária para screening. } \\
\text { Ligações telefônicas contadas, resultados dos testes de screening e } \\
\text { inquérito populacional sobre conhecimento de sífilis }\end{array}$ & Prevalência de sífilis & $\begin{array}{l}\text { Estudo } \\
\text { transversal }\end{array}$ \\
\hline Engelgau et al.; 1995.43 & $\begin{array}{l}\text { Campanha de intervenção em surto de sífilis, utilizando-se de redes } \\
\text { sociais como agente da intervenção, a partir de entrevistas de } \\
\text { contactantes. Resultados e custo comparados com um período sem } \\
\text { intervenção }\end{array}$ & $\begin{array}{l}\text { Incidência de sífilis, } \\
\text { análise de custo- } \\
\text { efetividade }\end{array}$ & $\begin{array}{l}\text { Estudo } \\
\text { transversal }\end{array}$ \\
\hline $\begin{array}{l}\text { Dubois-Arber et al.; } \\
1989.44\end{array}$ & $\begin{array}{l}\text { Avaliação da campanha "STOP-AIDS", iniciada na Suíça em 1987, } \\
\text { através de entrevistas e do monitoramento de venda de preservativos }\end{array}$ & $\begin{array}{l}\text { Estudos de prevalên- } \\
\text { cia }\end{array}$ & $\begin{array}{l}\text { Estudo } \\
\text { transversal }\end{array}$ \\
\hline Parsons et al.; 1991.45 & $\begin{array}{l}\text { Avaliação da implantação da Britsh Columbia AIDS Information On } \\
\text { Line, que foi acompanhada por campanha de mídia. Medida da } \\
\text { freqüência de ligações, do conhecimento adquirido com a campanha }\end{array}$ & Número de ligações & $\begin{array}{l}\text { Estudo } \\
\text { transversal }\end{array}$ \\
\hline Schooler et al.;46 & $\begin{array}{l}\text { Avaliação de diferentes veículos de comunicação em promoção de } \\
\text { saúde cardiovascular, através de entrevistas com participantes }\end{array}$ & $\begin{array}{l}\text { Impacto no conheci- } \\
\text { mento }\end{array}$ & $\begin{array}{l}\text { Estudo } \\
\text { transversal }\end{array}$ \\
\hline
\end{tabular}


Campanha de saúde + avaliação

\begin{tabular}{lll}
\hline Primeiro autor, & Metodologia utilizada & Medida do desfecho de estudo \\
ano de publicação & & Tipo de
\end{tabular}

Cristofolini et al.; 1993.47

Schou; 1987.48

Silveira et al.; 1999.49

Sogaard; 1988.50

Noronha; 1993.52

Min e Sander; 2003.5

Spira e Ayme; 1991.56

Chasan-Taber e

Tabachnick; 1999.54

Berry et al.; 1999.53

Naud et al.; 2000.55
Avaliação da campanha de detecção precoce do melanoma cutâneo em Trentino, através da comparação da mortalidade pelo melanoma em três cidades em que não ocorreu a intervenção

Avaliação da campanha de saúde oral em mídia de massa, voltada para crianças de cinco a sete anos e suas mães, com anúncios em revistas e TV e brochuras distribuídas nas escolas, através de entrevista com amostra de participantes

Avaliação da Campanha Nacional de Prevenção da Cegueira e Reabilitação Ocular, na região do $A B C$, onde 570 crianças foram triadas e 114 apresentaram baixa acuidade visual

Avaliação de campanha sobre doença periodontal, voltada para dentistas, na Noruega, promovendo educação continuada pela Associação de Dentistas Nacional, através de questionário enviado para uma amostra da população alvo

Avaliação de campanha de vacinação anti-meningocócica B da Secretaria Estadual de Saúde do Rio de Janeiro, em 1990, utilizando um estudo caso-controle

Pesquisa operacional do Projeto demonstrativo em epilepsia no Brasil, que pretende demonstrar, através de avaliação metodológica, que é possível estabelecer um modelo de atenção integral ao portador de epilepsia

Avaliação de quatro programas de intervenção em saúde, comparados com não intervenção

Avaliação da primeira campanha de prevenção de abuso sexual em crianças destinada ao público adulto, precedida de entrevistas para identificar facilitadores e barreiras, com inquérito populacional e entrevistas com autoridades e grupos focais

Avaliação de campanha de mídia sobre o papel do ácido fólico na prevenção dos defeitos do tubo neural na gestação, na China, comparando mulheres de duas áreas, uma de baixa e uma de alta incidência do agravo, que receberam prescrição de ácido fólico

Comparação da campanha de prevenção do câncer de colo uterino do Ministério da Saúde no Rio Grande do Sul em 1998 com as atividades de prevenção de rotina promovidas pela Secretaria Estadual de Saúde do Rio Grande do Sul.
Taxa de mortalidade Estudo específica transversal

Impacto no conhe- Estudo cimento transversal

Prevalência de baixa Estudo acuidade visual transversal

Impacto nas práticas Descrição de rotina qualitativa

Odds Ratio

Estudo caso-controle

Eficácia de interven- Pesquisa ção em serviço operacional

Incidência de agravos Estudo transversal

Impacto no conhe- Estudo

cimento transversal

Estudo observacional

Incidência de câncer Estudo de colo uterino transversal

SC = sífilis congênita; SINAN = Sistema Nacional de Agravos de Notificação; SIH = Sistema de Informações Hospitalares, VE = vigilância epidemiológica; $C D C=$ Centers for Disease Control and Prevention. DST= Doenças sexualmente transmissíveis. 


\section{Conclusões}

Os estudos de avaliação aqui colocados recorreram à epidemiologia descritiva ou aos métodos qualitativos para alcançar seus objetivos. Apenas um artigo que correspondeu às palavras-chave selecionadas se apresentava como uma pesquisa operacional. Não foram encontrados estudos que explicitassem claramente a tipologia de avaliação escolhida, como pesquisa avaliativa ou avaliação normativa ou formativa. O enfoque dado era o da análise de uma intervenção em saúde ou de uma campanha informativa (de mídia ou educativa) em seu curto prazo, ou seja, logo após a sua ocorrência, mostrando apenas os resultados imediatos. Não foi encontrada preocupação com a sustentabilidade da intervenção, não foram descritos indicadores ou condições traçadoras para uma formalização de estratégias e contabilização de resultados ou impacto de longo prazo.

A possibilidade de estabelecer um julgamento de valor da intervenção realizada, a partir da formalização de seus objetivos, suas atividades e seus resultados não foi o objeto principal dos trabalhos revisados, o que fortalece nossa argumentação inicial sobre a lacuna de conhecimento nesse campo de ação das políticas de saúde. Em nenhum caso encontramos a designação nem mesmo de indicadores de estrutura, processo e resultado, que apontasse ao menos para uma avaliação normativa, que poderia ocorrer no âmbito do programa de saúde que desenhara a intervenção.

\section{Referências}

1 Winslow CEA. The evolution and significance of the modern public health campaign. New Haven: Yale University Press; 1923.

2. Saraceni V, Leal MC. Avaliação da efetividade das campanhas para a eliminação da sífilis congênita no Município do Rio de Janeiro, 1999 e 2000. Cad Saúde Publica 2003; 19: 1341-9.

3. Ministério da Saúde. Programa Nacional de Doenças Sexualmente Transmissíveis e AIDS. Bases técnicas para eliminação da sífilis congênita. Brasília (DF); 1993.

4. Sífilis na gravidez. Saúde em Foco 1999; (23).

5. Saraceni V, Nicolai C, Domingues R, Lauria L, Durovni B, Boaretto MC. Sífilis adquirida e sífilis congênita: ações do município do Rio de Janeiro. DST: J Bras Doenças Sex Transm 2000; 12: 95 [Abstract].

6. Sífilis na gravidez. Saúde em Foco 2000; (24).

7. Singh AE, Romanowski B. Syphilis: review with emphasis on clinical, epidemiologic, and some biologic features. Clin Microbiol Rev 1999; 12: 187-209.
Campanhas podem ser instrumentos capazes de gerar modificações no estado de saúde de uma população. Com tal finalidade deveriam ser desenhadas e arquitetadas de modo a proporcionar os desdobramentos necessários para facilitar o acesso aos serviços e a conduzir à melhoria da saúde dos indivíduos. Para tanto, as campanhas deveriam fazer parte de atividades programáticas que seguissem um planejamento estratégico, com resultados de curto, médio e longo prazos esperados, medidos por indicadores claros e definidos por metas factíveis, que possibilitasse o julgamento de valor, produzindo mudanças no cenário da saúde pública. O monitoramento e a conseqüente avaliação dos resultados poderia levar a uma revisão dos programas e a modificações de rumo que se fizessem necessárias para trazer os ganhos esperados com os investimentos feitos.

A revisão realizada mostrou que existe um espaço a ser preenchido no campo da avaliação de campanhas de saúde, principalmente no que se refere à construção metodológica. Apesar de a palavra avaliação constar nesses vários artigos, a fundamentação teórica encontrada não foi condizente com as propostas apresentadas.

Muito se pode caminhar na trilha da avaliação, através de uma maior apropriação do conhecimento na área, integrando-se uma das metodologias já desenvolvidas ao desenho da intervenção de interesse, para que, a priori, sejam definidas as metas e os indicadores que propiciarão a avaliação do objeto.
8. Ramos-Filho C, May SB. Aspectos históricos das doenças sexualmente transmissíveis. Saúde em Foco 1998; 17: 5-11.

9. USDHEW (US. Department of Health, Education and Welfare). Syphilis: a synopsis. Atlanta; 2004.

10. Garnett GP, Aral SO, Hoyle DV, Cates W Jr, Anderson RM. The natural history of syphilis. Implications for the transmission dynamics and control of infection. Sex Transm Dis 1997; 24: 185-200.

11. Brown WJ, Donohue JF, Axnick NW, Blount JH, Ewe NH, Jones OJ. Syphilis and other venereal diseases. Cambridge; 1970.

12. Mahoney JF, Arnold RC, Harris AD. Penicillin treatment of early syphilis. Am J Public Health 1943; 33: 1387-91.

13. Carrara S. Tributo à Vênus: a luta contra a sífilis no Brasil, da passagem do século aos anos 40. Rio de Janeiro: FIOCRUZ; 1996.

14. Camargo ME. A sífilis avança progride o diagnóstico? Rev Assoc Med Bras 1988; 34: 19-23.

15. Fiumara NJ. Treatment of early latent syphilis of less than a 
year's duration: an evaluation of 275 cases. Sex Transm Dis 1978; 5: 85-8.

16. Pickering LK. Diagnosis and therapy of patients with congenital and primary syphilis. Pediatr Infect Dis 1985; 4 : 602-5.

17. Walker GJ. Antibiotics for syphilis diagnosed during pregnancy. Cochrane Database Syst Rev 2001; (3). [CD001143].

18. Ministério da Saúde. Programa Nacional de Doenças Sexualmente Transmissíveis. Testes de sensibilidade à penicilina: manual. Brasília (DF); 1999.

19. CDC (Centers for Disease Control and Prevention). Sexually transmitted diseases: treatment guidelines. Atlanta; 2002. [Report n. 51].

20. Tayra A. O sistema de vigilância epidemiológica da sífilis congênita no Estado de São Paulo, 1989-1997 [dissertação de mestrado]. São Paulo: Faculdade de Saúde Pública da Universidade de São Paulo; 2001.

21. Silva Jr. JB, Mendes ACG, Campos Neta TJ, Lyra TM, Medeiros KR, Sá DA. Sistema de informações hospitalares - fonte complementar na vigilância e monitoramento de doenças transmitidas entre pessoas. Inf Epidemiol SUS 2000; 9: 137-64.

22. Gomes de Haz H, Farias Reinoso AT. Evaluación de la vigilancia de la sífilis congênita em Ciudad de la Habana. Rev Cub Salud Publica 1998; 24: 73-7.

23. Martin D, Bertrand J, McKegney C, Thompson L, Belongia E, Mills W. Congenital syphilis surveillance and newborn evaluation in a low-incidence state. Arch Pediatr Adolesc Med 2001; 155: 140-4.

24. CDC (Centers for Disease Control and Prevention). Evaluation of congenital syphilis surveillance system - New Jersey, 1993. MMWR Morb Mortal Wkly Rep 1995; 44: 225-7.

25. Cohen DA, Scribner R, Cory D. Controlling a syphilis epidemic. West J Med 1992; 157: 430-2.

26. Mascola L, Pelosi R, Blount JH, Alexander CE, Cates WJr. Congenital syphilis revisited. Am J Dis Child 1985; 139: 575-80.

27. Alexander JM, Sheffield JS, Sanchez PJ, Mayfield J, Wendel GDJr. Efficacy of treatment for syphilis in pregnancy. Obstet Gynecol 1999; 93: 5-8.

28. McFarlin BL, Bottoms SF. Maternal syphilis in Michigan: the challenge to prevent congenital syphilis. Midwifery 1995; 11: 55-60.

29. Phaosavasdi S, Snidvongs W, Thasanapradit P, Ungthavorn $\mathrm{P}$, Bhongsvej $\mathrm{S}$, Jongpiputvanich $\mathrm{S}$, Vesjajiva $\mathrm{S}$, Udomsantisuk P, Chitwarakorn A, Panikabutra K. Effectiveness of benzathine penicillin regimen in the treatment of syphilis in pregnancy. J Med Assoc Thai 1989; 72: $101-8$.

30. Coles FB, Muse AG, Hipp SS. Impact of mandatory syphilis delivery test on reported cases of congenital syphilis in Upstate New York. J Public Health Manag Prac 2004; 4: 50-6.

31. Fitzgerald DW, Behets FM, Lucet C, Roberfroid D. Prevalence, burden, and control of syphilis in Haiti's rural Artibonite region. Int J Infect Dis 1998; 2: 127-31.
32. Garland SM, Kelly VN. Is antenatal screening for syphilis worth while? Med J Aust 1989; 151: 368, 370, 372.

33. Swingler GH, Van Coeverden de Groot HA. The antenatal prevention of congenital syphilis in a peri-urban settlement. S Afr Med J 1993; 83: 34-5.

34. Fitzgerald DW, Behets F, Preval J, Schulwolf L, Bommi V, Chaillet P. Decreased congenital syphilis incidence in Haiti's rural Artibonite region following decentralized prenatal screening. Am J Public Health 2003; 93: 444-6.

35. Hayes R. Improved STD treatment: a message of hope. Special report. Tanzania. AIDS Anal Africa 1995; 5: 10-1.

36. Herdorn N. Can "mass treatment" work with STDs? Network 1992; 12: 12-3.

37. Perez-Stable EJ. The AIDS epidemic in Cuba. Rev Panam Psicol 1992; 24: 71-84.

38. Bentley EM, Holloway PJ. An evaluation of the role of health visitors in encouraging dental attendance of infants. Comm Dent Health 1993; 10: 243-9.

39. Card JJ. Teen pregnancy prevention: do any programs work? Annu Rev Public Health 1999; 20: 257-85.

40. Chen JL, Kodagoda D, Lawrence AM, Kerndt PR. Rapid public health interventions in response to an outbreak of syphilis in Los Angeles. Sex Transm Dis 2002; 29: 277-84.

41. Storey D, Boulay M, Karki Y, Heckert K, Karmacharya DM. Impact of the integrated Radio Communication Project in Nepal, 1994-1997. J Health Commun 1999; 4: 271-94.

42. Towpik J. Critical evaluation of the venereal disease control campaign in Poland 1970-76. Br J Vener Dis 1979; 55: 127 30.

43. Engelgau MM, Woernle CH, Rolfs RT, Greenspan JR, O'Cain M, Gorsky RD. Control of epidemic early syphilis: the results of an intervention campaign using social networks. Sex Transm Dis 1995; 22: 203-9.

44. Dubois-Arber F, Lehmann P, Hausser D, Gutzwiller F. Evaluation of campaigns for the prevention of AIDS in Switzerland in 1987. Rev Epidemiol Santé Publique 1989; 37: 207-16.

45. Parsons DC, Bell MA, Gilchrist LD. Evaluation of the British Columbia AIDS Information Line. Can J Public Health 1991; 82: 399-403.

46. Schooler C, Chaffee SH, Flora JA, Roser C. Health campaign channels: tradeoffs among reach, specificity, and impact. Hum Commun Res 1998; 24: 410-32.

47. Cristofolini M, Bianchi R, Boi S, DeCarli A, Micciolo R, Cristofolini P, Zumiani G. Effectiveness of the health campaign for the early diagnosis of cutaneous melanoma in Trentino, Italy. J Dermatol Surg Oncol 1993; 19: 117-20.

48. Schou L. Use of mass-media and active involvement in a national dental health campaign in Scotland. Comm Dent Oral Epidemiol 1987; 15: 14-8.

49. Silveira RC, Villano DM, Interlandi R, Rehder JRCL. Veja bem Brasil: oftalmologia preventiva. Arq Med ABC 1999; 22: 47-9.

50. Sogaard AJ. Effect of a nationwide periodontal health campaign on dentists' awareness, attitude and behavior in Norway. Comm Dent Oral Epidemiol 1988; 16: 30-5. 
51. Min LL, Sander JWA. Projeto demonstrativo da epilepsia no Brasil. Arq Neuropsiquiatr 2003; 61: 153-6.

52. Noronha CP. Avaliação da eficácia da vacina antimeningocócica BC no Rio de Janeiro: um estudo caso-controle [dissertação de mestrado]. Rio de Janeiro: Escola Nacional de Saúde Pública da Fundação Oswaldo Cruz; 1993.

53. Berry RJ, Li Z, Erickson JD, Li S, Moore CA, Wang H, Mulinare J, Zhao P, Wong LY, Hong SH, Correa A. Prevention of neural-tube defects with folic acid in China. China-US Collaborative Project for Neural Tube Defect Prevention. N Engl J Med 1999; 341: 1485-90.
54. Chasan-Taber L, Tabachnick J. Evaluation of a child sexual abuse prevention program. Sex Abuse 1999; 11: 279-92.

55. Naud P, Matos JC, Hammes L, Vettorazzi J. Avaliação dos programas de screening para o câncer de colo uterino no estado do Rio Grande do Sul. Rev Hosp Clin Porto Alegre 2000; 20: 108-13.

56. Spira N, Ayme S. Epidemiological evaluation of four public health interventions. Rev Epidemiol Santé Publique 1991; 39 [Suppl 1]: S75-S82.

Recebido em 21 de dezembro de 2004

Versão final em 13 de maio de 2005

Aprovado em 3 de agosto de 2005 